\title{
¿Es la ciudad de México policéntrica? Nuevos datos y algoritmos para la detección de centralidades urbanas
}

\section{Is Mexico city polycentric? New data and algorithms for urban centralities detection}

\author{
Jorge Alberto Montejano-Escamilla*
}

\begin{abstract}
Abtsract
With the emergence of new algorithms for network analysis and the development of economic databases disaggregated at a unit level, I propose a method different from those previously reported in the literature to detect urban centralities.

The first part defines urban centrality and presents a theoretical debate on the polycentric condition. The second part shows previously used methods for measuring centralities and describes the new tools used in this research. Finally, we present some results from this method applied as a case study in Mexico City.
\end{abstract}

Keywords: Network analysis, urban centrality, Mexico city.

\section{Resumen}

Con la emergencia de nuevos algoritmos para el análisis de redes y la aparición de bases de datos económicas desagregadas a nivel de unidad, se plantea un método distinto a los anteriormente reportados en la literatura especializada para detectar centralidades urbanas.

La primera parte define la centralidad urbana y presenta una disertación teórica sobre la condición policéntrica. Posteriormente, se muestran los métodos anteriormente usados para la medición de las centralidades y se describen las nuevas herramientas empleadas en esta investigación. Finalmente, se presentan algunos resultados de este método aplicado como caso de estudio en la ciudad de México.

Palabras Clave: Análisis de redes, centralidad urbana, ciudad de México.

* Centro de Investigación en Geografía y Geomática “Ing. Jorge. L. Tamayo”, A. c. (CentroGeo). Correo-e: jmontejano@centrogeo.org.mx 


\section{Introducción}

El presente artículo pone a consideración una nueva metodología para la detección de centralidades urbanas, basada en la utilización de geodatos de unidades económicas en conjunción con algoritmos desarrollados por investigadores del Instituto de Tecnología de Massachusetts.

A diferencia de los métodos anteriormente reportados en la literatura, donde los procesos de detección se basan principalmente en la densidad de empleos por unidad de medida, el propuesto supone una distinta mirada a la definición de centralidad, tanto en lo referente al cómo medirla, como en lo relativo a su relación con la forma edificada. Asimismo, mientras que para otros procedimientos la red vial le es indiferente, para el que se propone es un elemento esencial que posibilita la medición de centralidades a través del análisis espacial y de la interacción entre dicha red y las unidades económicas.

El documento se estructura en tres partes. La primera es una revisión acrítica de la literatura referente a los conceptos de centro, centralidad y policentrismo, que sirve de marco teórico para encuadrar la presente investigación. La segunda, se centra en algunos de los métodos más importantes de medición de centralidades reportados por la literatura, destacando los rasgos principales y esbozando algunas diferencias con el método a proponer.

Además, se hace una descripción detallada sobre el método propuesto, resaltando su innovación en términos del uso de la información para lograr el objetivo planteado. Finalmente, se describen algunos de los resultados más relevantes, destacando las limitaciones de este ejercicio sobre la ciudad de México, principalmente debido a la disponibilidad de los datos al momento de realizar esta investigación, y proponiendo su ampliación como método válido para detectar centralidades en un ámbito metropolitano.

\section{Centro y centralidad}

El concepto de centralidad urbana alberga al menos tres dimensiones bien definidas; ${ }^{1}$ En primer lugar, puede referirse a un tipo de localización del espacio (centro geográfico). La segunda (la dimensión social e histórica)

\footnotetext{
${ }^{1}$ Quizá la definición que más se acerca a nuestra noción esté contenida en esta cita: "Para el urbanista medio, el centro es una parte de la ciudad delimitada espacialmente (por ejemplo, situada en la confluencia de un esquema radial de vías de comunicación) que desempeña una función a la vez integradora y simbólica. El centro es un espacio debido a las características de su ocupación, permite una coordinación de las actividades urbanas, una identificación simbólica y ordenada de estas actividades y, por consiguiente, la creación de las condiciones necesarias a la comunicación entre actores" (Castells, 1974: 263).
} 
tiene que ver con "un conjunto de funciones y de grupos sociales localizados sobre un lugar de características más o menos específicas" (Casado, 2010). Un tercer tipo de centro (económico) es aquél donde se concentra una buena parte de los bienes y servicios producidos o consumidos dentro de una ciudad o región. Ésta noción de centralidad es entendida por Castells (1974: 262) "como la organización espacial de los puntos clave en que se desarrollan las diferentes fases del proceso del intercambio entre los procesos de producción y consumo".

El vocablo de centro, originario del latín centrum, ya no sirve solamente para "indicar un punto en el plano, sino también una posición en el espacio, y adquiere caracteres desvinculados de la geometría y basados en aspectos funcionales..." (Paris, 2013: 48). Este autor sugiere que dado que el territorio de hoy es muy distinto al que se estudiaba hasta los años setenta, es necesario utilizar el término de "lugar de centralidad" en contraposición al centro, para describir los espacios donde actualmente se aglutinan las funciones centrales. A este respecto, Terrazas (2004: 235) sugiere que en la discusión sobre centro y centralidad es indispensable primero determinar las funciones y actividades que se realizaban en el "centro inicial" y que ahora encuentran localización también fuera de él, y las actividades derivadas principalmente de las dinámicas sociales ligadas a la transformación de la base económica mundial, que no necesariamente se localizan en el centro inicial, sino que de hecho, muchas de ellas se asientan en las periferias.

Mientras que para Terrazas (2010: 9) "El concepto de centro [...] se refiere al lugar en el territorio urbano donde se realizan las actividades sociales más intensas como son el comercio, los servicios, las manifestaciones culturales y políticas y, en general, el intercambio social más significativo", para González (2010: 30) "La acepción [...] de la noción de centralidad es la propiedad que tienen algunos lugares de polarizar el espacio, de su capacidad de atracción de personas, objetos, funciones e información”.

Las anteriores definiciones son complementarias, pues mientras que la primera supone que para que haya un intercambio significativo es imprescindible la presencia de personas, la segunda asume que las propias actividades generadas son suficiente motor para atraer personas. En cualquier caso, la interacción entre actividades y personas es el elemento esencial de la noción de centralidad. ${ }^{2}$

${ }^{2}$ El propio Castells (1974) argumenta que uno de los elementos más importantes en una centralidad urbana es la "creación de las condiciones necesarias a la comunicación entre actores". En el mismo sentido, diversos investigadores, dedicados a explicar porqué sigue existiendo una alta capacidad de atracción de las fuerzas de aglomeración en momentos en que los costes de transporte continúan reduciéndose (Camagni, 2005; Storper y Venables, 2002), argumentan que son las relaciones cara a cara uno de los elementos clave para entender ese fenómeno. 
A pesar de que Terrazas argumenta que una centralidad no es resultado únicamente de una acumulación de bienes inmobiliarios, sí constituye una característica adicional de las centralidades: una mayor densidad de actividades, la cual generalmente va acompañada por una mayor densidad de personas. ${ }^{3}$

Dicha densidad relativa de actividades y personas nos remite al concepto de aglomeración o clúster, término definido por Porter (1998) como "una masa crítica de compañías en una localización particular (un país, estado, región o incluso una ciudad)". Según Mercado y Moreno (2011), el concepto de clúster incluye una dimensión adicional relativa a la especialización, entendiéndolos como "concentraciones geográficas de compañías e instituciones interconectadas en un campo particular" (Porter, 1998 en Mercado y Moreno, 2011).

Para Camagni (2005), las aglomeraciones en los centros económicos se caracterizan por conformar clústeres bajo el principio de sinergia, resultado de una maximización de beneficios relacionados con la disminución de los costes de producción y el aumento de la renta mediante un modo homogéneo de producción a gran escala (economías de escala) que permiten, por su tamaño y proximidad, disminuir el costo por unidad producida. El límite de estas aglomeraciones está dado básicamente por los costes del transporte y, en un segundo término, por los costes crecientes o deseconomías derivados de la misma aglomeración. ${ }^{4}$

Pero no solamente la producción busca la reducción de costes y aumento de los beneficios mediante el asentamiento contiguo. Determinado tipo de comercios y servicios buscan localizaciones por complementariedad, intentando aprovechar la atracción generada por localizaciones previas y asentadas con éxito (Kunz, 2003). En este sentido, las centralidades generalmente implican un asentamiento de clústeres complementarios (oficinas, restaurantes, etcétera).

Mientras que el principio de sinergia explica la tendencia de las unidades económicas a agruparse, el principio de accesibilidad (o de competencia espacial) explica las lógicas de localización. Éste supone que existe una barrera espacial (fricción) para la movilidad de bienes y personas (costes de transporte, tiempo) y para el intercambio de servicios e información. ${ }^{5}$ El grado de accesibilidad determina la lógica de localización individual

${ }^{3}$ Consideramos por ello que las aglomeraciones productivas (manufactura) no deben formar parte del análisis, ya que a pesar de atraer personas, la naturaleza actual de la actividad no implica la búsqueda de una situación central y no implica una mezcla de actividades.

${ }^{4}$ Weber y Friedrich (1929) preveían una localización aglomerada de las unidades productivas sólo cuando las ventajas de la aglomeración y los ahorros superaran a los costes de transporte ocasionados por una determinada localización.

${ }^{5}$ El concepto de impedancia espacial fue planteado inicialmente por Haig (1926), entendiendo que sin ésta no habría costes de transporte (Graizbord, 2008: 57). 
de los actores económicos, que "a su vez dan lugar, por efectos acumulativos, a la estructuración de todo el espacio, tanto a nivel macro como a nivel microterritorial "(Camagni, 2005: 51). Este principio, que parte del modelo teórico de von Thünen para explicar la localización de las actividades agrícolas, gobierna no sólo a los agentes económicos sino también las decisiones residenciales.

El concepto es que la localización óptima urbana de las actividades productivas (empresa) debe de encontrarse en el punto de tangencia que permita obtener el beneficio más elevado y la renta de suelo más baja. Richard M. Hurd (1905) citado en (Alonso, 1964: 6) señaló que: "dado que el valor (del suelo urbano) depende de la renta, y la renta de la localización, y la localización de la conveniencia, y la conveniencia de la cercanía, podemos eliminar todos los pasos intermedios y decir que el valor depende de la cercanía". Esto explicaría, en un modelo teórico y monocéntrico, la tendencia a determinadas empresas a localizarse lo más cercano posible al mercado potencial (el cual históricamente coincidía con el centro tradicional) así como la presencia de clústeres dentro de las centralidades urbanas. Por lo anterior, la detección de clústeres complementarios se convierte en un indicador fiable de mezcla de actividades y personas, definición que nosotros asumimos como una centralidad urbana.

\section{Policentrismo y descentralización}

Aun cuando las centralidades urbanas de tipo económico estuvieron en un inicio íntimamente ligadas con la traza urbana y la forma edificada — coincidiendo generalmente la localización del centro económico con el centro histórico en las primeras etapas del desarrollo de una ciudad-, éstas han emergido en la actualidad dentro del territorio sin guardar una lógica consecuente con el principio reinante hasta antes del inicio de la era postindustrial, que era la de localizarse cerca de los mercados. ${ }^{6}$

Las hipótesis económicas para explicar la emergencia de nuevas centralidades se fundamentan en varios estudios, entre los que destacan los de Allen (1997), Fujita y Mori (1997) y Fujita et al. (2001), que plantean que - en un inicio- existen ventajas en producir y consumir concen-

\footnotetext{
${ }^{6} \mathrm{El}$ arquetipo de ciudad industrial encuentra su declive como modelo hacia 1940 y su declive como realidad física a mediados de los ańos setenta. La crisis mundial de las energías de 1973 obliga a los países desarrollados a replantear su sistema de producción, observándose una desconcentración de la industria a favor de los países emergentes a la par que se observaba el fenómeno de tercerización económica detectado por Colin Clark (Hall, 1997). Esta transformación en el modelo de producción (de uno Fordista a uno flexible) produjo una reducción en el volumen de las factorías madre y con ello sobrevino un proceso de externalización de varias fases del proceso productivo (outsourcing), permitiendo en parte la emergencia de una serie de empresas que se fueron asentando donde el suelo era barato y coadyuvando a la creación de subcentros de empleo.
} 
tradamente; en una siguiente etapa, la concentración (hiper-concentración) genera deseconomías que a largo plazo terminan por destruir las cualidades originales de la centralidad provocando la dispersión y la emergencia de nuevas centralidades. ${ }^{7}$

Esto es apoyado por teóricos como White (1999), quien señala que las deseconomías de aglomeración, es decir, la congestión y los elevados precios del suelo en un área central, actúan como fuerzas centrífugas que expulsan parte de la actividad económica (Muñiz et al., 2005).

Adicionalmente, se argumenta que uno de los orígenes del policentrismo, desde el marco teórico del modelo de ciudad central, se debe a la caída en los costes de transportes, permitiendo la posibilidad de que las ciudades se especialicen en algún sector cubriendo un área de mercado más extensa. Dichas economías de aglomeración, surgidas de la especialización (economías de localización), al parecer adquieren ventajas al situarse estratégicamente en la región debido a una sinergia creada entre aglomeraciones complementarias (Camagni, 2005; Dematteis, 1994) tendente a configurarse en forma de redes.

El policentrismo ha sido también categorizado desde dos puntos de vista: Clark y Kuijpers-Linde (1994) y Champion (2001), citados en Muñiz et al. (2005). El primero acepta la posibilidad de la coexistencia de diferentes centros de empleo (subcentros descentralizados); el segundo señala una integración de las áreas de mercado de los núcleos próximos que en el pasado habían evolucionado con una cierta autonomía (subcentros integrados). Mientras que en la primera situación los subcentros de empleo pudieran estar representados por poblaciones cercanas al núcleo central original, el segundo escenario plantea la integración de ellos al núcleo central bajo un esquema espacial de crecimiento por conurbación.

\section{Métodos para medir centralidades}

Aun cuando existen diversos puntos de vista (geográfico, económico, etc.), parece ser que el objetivo primario en la caracterización de las centralidades es la organización de las actividades en el territorio, de modo que la toma de decisiones reduzca los costos y maximice los beneficios (econó-

\footnotetext{
${ }^{7}$ Este hallazgo guarda relación con el modelo de R. Vernon que explica el ciclo productivo de las empresas y su localización en el territorio. Según esta teoría, los sectores productivos tienden a modificar su localización territorial dependiendo del estado o fase de su ciclo de vida. En un inicio, se asientan en el centro y en la semiperiferia; durante el crecimiento se expanden hacia la periferia pero con mayor intensidad en el centro; en la fase de madurez se observa una concentración mayor en la periferia (disponibilidad de más suelo más barato) y en la fase de declive se decantan en su mayoría por una mayor densidad en la periferia (Méndez y Caravaca, 1996).
} 
micos, sociales, medioambientales). ${ }^{8}$ La detección de centralidades en distintas escalas (urbana, metropolitana, regional, nacional y mundial) permite la abstracción de la complejidad que representan los asentamientos humanos y con ello generar modelos que permitan la racionalización de los recursos.

Las aplicaciones puntuales de la detección de centralidades varía dependiendo de la escala, la búsqueda de equilibrio entre los distintos usos, podría ser en la urbana; sucitándose la localización idónea de un negocio en una rama específica o la inclusión de equipamientos públicos donde no los hay; en la metropolitana, la consolidación de corredores de desarrollo o polígonos de actividades; en la regional, la jerarquización de las poblaciones para la dotación de infraestructuras hospitalarias; en la nacional, la distribución y jerarquía de la red de carreteras y autopistas, y en la mundial, la división internacional del trabajo, especialización productiva y focalización de inversión extranjera directa.

Ahora bien, como señala Terrazas (2010), la centralidad tiene que ser medida con relación a las personas. Son ellas quienes construyen las centralidades de manera cotidiana. Pudiera medirse la centralidad simplemente en términos geométricos o cuantificando la masa edilicia de una ciudad o región en cuestión, pero la medición no tendría mucho sentido por sí sola para la planeación, puesto que son la densidad de personas y actividades lo que interesa para dar sentido al territorio. ${ }^{9}$

Así, el principal indicador tradicionalmente utilizado para medir espacios centrales ha sido el empleo, variable que involucra directamente tanto actividades como personas, e indirectamente, los flujos que las personas generan al desplazarse hacia sus centros de trabajo.

Dentro de esta corriente de métodos de detección de subcentros de empleo como indicador de policentrismo aparecen los trabajos de Bogart y Ferry (1999), quienes definen a los centros como lugares con alta den-

\footnotetext{
${ }^{8}$ Castells (1974: 264) argumenta que "Cambiar el marco vital es cambiar las relaciones sociales [...] los planes de urbanismo, suscitados generalmente por el deseo de frenar la 'desorganización social urbana’, están animados por un espíritu reformador, y por tanto, integrador. Allí donde se constata ruptura de las relaciones sociales o débil interiorización de los valores dominantes, hay que crear un polo integrador [...] [cuyas] [...] principales características [sean] la concentración de las actividades destinadas a favorecer la comunicación [y la] accesibilidad con respecto al conjunto de la zona urbana que se asume la centralidad".

${ }^{9}$ Existen herramientas informáticas diseñadas exprofeso para la detección de centralidades urbanas, como la desarrollada dentro de la University College London (UCL) denominada DepthMap (parte del módulo de Space Syntax). Dichas herramientas son muy útiles para la promoción de nuevas centralidades en términos de la morfología urbana. Sin embargo, esta fue diseñada exprofeso para que los arquitectos pudieran observar el efecto del diseńo del edificio en las personas. A pesar de que su uso se ha ampliado a ámbitos como la planeación urbana o la criminología, no es una herramienta que incluya las cualidades del edificio como medida de accesibilidad. Por otra parte, existe también un modelo denominado lisa (Local Indicators of Spatial Association), el cual es un modelo estadístico espacial que busca aglomeraciones basadas en valores similares.
} 
sidad y alta cantidad de empleo; el de Thurstain-Goodwin y Unwin (2000), quienes localizaron centros en poblaciones usando estimaciones de densidad de Kernel, y el de Batty (2001), quien destacó la emergencia de ciudades policéntricas mediante el uso de simulación de agentes (Suárez y Delgado, 2009). Dentro de esta misma línea, encontramos el trabajo de Muñiz et al. (2005) y García-López y Muñiz (2010) quienes, mediante el análisis de la densidad del empleo, generan jerarquías para así determinar la condición polinuclear de la región metropolitana de Barcelona.

Entre los métodos más utilizados con relación a datos de empleo (citados en Muñiz et al., 2005 y en Suárez y Delgado, 2009) se encuentran el método McDonald (1987), el cual escoge las unidades administrativas donde la densidad bruta de empleo sea mayor a la de municipios adyacentes; y el método Giuliano y Small (1991), que funciona mediante la creación de umbrales mínimos para la densidad y el total de puestos de trabajo (los tramos urbanos que lo sobrepasen son identificados como potenciales subcentros).

Para el caso de la ciudad de México, Garrocho (1996) determinó el policentrismo a partir de la distribución de la población en el territorio derivada de datos residenciales; Aguilar y Alvarado (2005) determinaron un umbral mínimo de empleo (al menos 5,500 por unidad de medida) para determinar los subcentros; Graizbord y Acuña (2005) han propuesto un análisis de los centros utilizando flujos sobresalientes (datos de movilidad intermunicipales provenientes de las encuestas origen-destino), ${ }^{10}$ mientras que Suárez y Delgado (2009) identificaron subcentros basados tanto en concentración de empleo como en capacidad de atracción de viajes, utilizando para ello datos provenientes principalmente del censo económico y del de población.

Una de las investigaciones recientes más destacadas es la realizada por Salomón González (2010), quien a partir del análisis de datos de movilidad (encuesta origen-destino) para algunos municipios de la Zona Metropolitana del Valle de México (zMvm) y su modelación espaciotemporal, logra resultados que, además de ser muy similares a los obtenidos por Suárez y Delgado (2009) en términos espaciales, agregan un valor adicional a la detección de dichas centralidades: la dimensión temporal en la cual ocurren las actividades no residenciales.

${ }^{10}$ Esta línea de exploración de centralidades partiendo de los flujos ha sido trabajada también por Hall y Pain (2006), quienes hicieron un estudio de varias regiones metropolitanas europeas en busca de un patrón común que las pudiera identificar a todas ellas como mega regiones policéntricas, utilizando para ello datos provenientes de intercambio de correos electrónicos, vuelos aéreos, movilidad obligada, llamadas telefónicas, etc. El final no llega a ser concluyente en términos de la condición policéntrica, debido a que se observó que la mayoría de las empresas de tecnología avanzada buscan localizaciones céntricas en ciudades de primer orden debido a la presencia de mano de obra calificada. 
González (2010: 31) argumenta que, a diferencia de la geografía histórica, la geografía-tiempo "desarrolla una perspectiva innovadora al interesarse en el comportamiento de las acciones humanas en el espacio y tiempo". Mediante el uso de diversos modelos desarrollados por la geografía-tiempo (la trayectoria espaciotemporal, el prisma espaciotemporal y la noción de estación) que permiten determinar lugares donde dos o más trayectorias (viajes) concurren en espacio y tiempo, y en conjunción con el reconocimiento del pico de las actividades no residenciales, González (2010) logra identificar centralidades predominantes y su duración mediante la integración de los datos en un Sistema de Información Geográfica y su interpolación para finalmente generar una imagen continua de actividades. El resultado sería similar al de observar a un conjunto de bombillas de un árbol de navidad encenderse y apagarse, (cada una con distinta intensidad) en diferentes momentos del día.

Probablemente, lo que resulta más interesante de este método es que se asume a priori la prexistencia de clústeres de actividad económica debido a la concentración de flujos en un espacio determinado (estación), mientras que en el método tradicional, se asume la situación inversa donde la densidad de empleo implica la pre-existencia de flujos a ese punto de convergencia.

\section{Nuevos algoritmos e información disponible para la detección de centralidades}

Ya en su investigación, Suárez y Delgado (2009) apuntaban que no existía hasta la fecha de publicación de su análisis, intentos por identificar subcentros dentro de la ciudad de México usando mapas de usos del suelo. Argumentaron que sería muy útil pues se podrían seleccionar usos comerciales, industriales y de oficinas con alta densidad.

En este sentido, se presenta ahora la coincidencia temporal para poder llevar a cabo este tipo de estudio: el desarrollo de nuevos algoritmos para la detección de centralidades y, por otro lado, la publicación en línea de una base de datos que contiene una gran cantidad de unidades económicas georeferenciadas para la ciudad de México.

Andrés Sevtsuk y Michael Mekonnen (2001), del grupo de investigación de la forma de la ciudad del Instituto Tecnológico de Massachusetts (мIт), han desarrollado recientemente (2011) una serie de algoritmos cuyo objetivo es el de poder realizar análisis de redes urbanas, condensados en forma de una herramienta modular que funciona en conjunto con el software ArcGis. A diferencia de las anteriores herramientas existentes en el mercado, y cuya característica principal es la de operar con dos elementos — vértices y aristas (o nodos y arcos)—, la herramienta de 
análisis de centralidades urbanas de Sevtsuk y Mekonnen incluye un tercer elemento en el estudio: el edificio.

Los autores argumentan que la mayoría de los estudios de redes habían utilizado solamente nodos y arcos, ${ }^{11}$ resultando insuficientes para explicar determinados fenómenos urbanos en los que los edificios - los cuales albergan la mayor parte de las actividades donde comienzan y terminan la mayor parte de los viajes - desempeñan un papel preponderante.

Mientras que un estudio que involucra solamente nodos y arcos (y que toma a los arcos como unidad de análisis) devolverá valores idénticos para todos los edificios que estén localizados sobre el arco en cuestión -no importando si un edificio está localizado en una orilla o en la parte central de la calle_- el modelo que incorpora al edificio permite obtener resultados diferenciados dependiendo de su localización espacial y la relación que guarde con el arco, incorporando el valor que comporta la situación de los edificios en el entorno real construido.

Adicionalmente, este edificio puede ser evaluado dentro del conjunto con una ponderación diferenciada del resto de nodos, dependiendo de su importancia en el contexto, ya sea porque es una construcción icónica, por su valor en el mercado, por población residente o simplemente por el número de niveles.

Así, la herramienta UNA (Urban Network Analysis) utiliza arcos, las cuales representan el camino o calles por las que un transeúnte puede viajar; nodos, los cuales representan la intersección entre una o más arcos en el espacio; y edificios, pudiendo ser representados ya sea mediante un polígono o un punto y los cuales se convierten en la medida de análisis.

Se asume de entrada que los edificios están conectados a las calles que se encuentran en la distancia perpendicular más corta a la localización de los edificios. De este modo se simula la distancia mínima que alguien tendría que recorrer desde la salida principal del edificio a su calle adyacente.

Esta herramienta de análisis realiza cálculos de centralidad mediante mediciones basadas en "métodos matemáticos que cuantifican la importancia de cada nodo en una gráfica...” (Sevtsuk y Mekonnen, 2011: 4). Esto es, mide qué tan céntrico es cada elemento computado con respecto a los otros elementos adyacentes. La herramienta UNA computa cinco distintos tipos de métrica de centralidad: Alcance (Reach), Índice de Gravedad (Gravity Index), Intercesión (Betweenness), Cercanía (Closeness)

\footnotetext{
${ }^{11}$ Probablemente la herramienta de análisis que más se acerque a la desarrollada por Svetsuk y Mekonnen sea Sanet (Spatial Analyst Along Networks), la cual es capaz de analizar eventos que ocurren en y a lo largo de la red. La herramienta realiza funciones de análisis como diagramas de Voronoi o estimaciones de densidad Kernel, entre otras. Sin embargo, no se observa que la herramienta desarrollada utilice al edificio como tercer elemento para su análisis, o al menos, no lo hace explícito. Para mayor detalle ver Okabe et al., 2006.
} 
y Rectitud (Straightness). ${ }^{12}$ Cada uno de ellos puede ser computado con variables restrictivas (radio de distancia específico) y una ponderación que le daría mayor o menor peso a cada elemento computado, dependiendo del análisis en cuestión. A continuación se describen brevemente cada una de las herramientas:

\subsection{Alcance (Reach)}

Describe el número de edificios (puntos o polígonos) en un radio de búsqueda determinado, que son asequibles desde un edificio específico en la distancia más corta posible a través de la red vial. La principal utilidad es corroborar la existencia de clústeres, centralidades consolidadas o en proceso de formación. Ello se logra debido a la categorización que el algoritmo realiza y que depende del número de edificios alcanzables desde cada edificio en un radio previamente especificado.

Este algoritmo se puede utilizar para conocer, por ejemplo, cuantas locaciones son accesibles dentro de una distancia caminable (radio de $400 \mathrm{~m}$ ) desde un punto de partida. Es una herramienta muy potente que podría determinar, por ejemplo, dentro de un plan de peatonalización, las calles que podrían ser sujetas a restringir el uso automotor para enfatizar una centralidad peatonal. Permite determinar también las necesidades presentes y futuras tanto de transporte como de equipamientos urbanos, entre otras conclusiones posibles.

Si a cada edificio computado se le asigna un peso específico (número de empleados, por ejemplo), el resultado arrojado será la suma de los pesos específicos en lugar del número de destinos.

\subsection{Indice de Gravedad (Gravity Index)}

El índice de gravedad realiza la misma acción que el Alcance, con la diferencia de añadir factores adicionales de impedancia para alcanzar a los diferentes edificios. Estos factores de resistencia, que actuarían como barreras u obstáculos para alcanzar un destino desde un punto determinado, fueron primeramente descritos por Hansen (1959) e incluyen conceptos como la distancia, el precio del viaje o incluso, el tráfico existente en una vía como factor negativo. Esta herramienta es utilizada comúnmente como una medida de accesibilidad en la planeación del transporte.

\footnotetext{
${ }^{12}$ Para revisar a fondo cada algoritmo de métrica de centralidad, ver Sevtsuk y Mekonnen, 2001.
} 


\subsection{Intercesión (Betweenness)}

Es una función que puede ser utilizada para estimar la cantidad de tráfico peatonal que una locación recibe. Este indicador es interesante en el sentido en el que puede ser utilizado para determinar el éxito o fracaso de localización de un negocio dependiendo del flujo de personas que transita con destino a ese negocio o que tiene que pasar frente a él para llegar a otro destino. Originalmente utilizada como una medida de centralidad en materia de comunicación, ha resultado muy útil en estudios urbanos.

La Intercesión de un edificio determinado estima el número de ocasiones en que este edificio recae en los caminos más cortos entre pares de otros edificios alcanzables dentro de un radio determinado. ${ }^{13}$

Si esta función es ponderada por algún tipo de característica demográfica, por ejemplo, por población con recursos económicos mayores, el resultado capturaría el potencial de transeúntes con alto poder adquisitivo que transitan por un edificio específico en su camino a ese u otro destino.

\subsection{Cercania (Closeness)}

Esta función es una medida de cuan central es un lugar, determinado por los caminos más cortos para unir nodos más cercanos, lo que ocurre en las intersecciones de las calles. Indica que tan cerca están cada una de las locaciones con respecto a todas las demás locaciones circundantes dentro de un umbral o radio determinado, viajando a través de la red vial.

Permite la determinación de centralidades, tanto en áreas urbanas como en zonas metropolitanas, basadas en la relación geométrica que guardan los elementos que conforman el sistema (nodos, arcos, edificios). Este tipo de medida permite plantear nuevas especializaciones económicas en zonas cuyas características morfológicas denoten una centralidad geométrica.

\subsection{Rectitud (Straightness)}

Describe en qué medida, rutas de un lugar a otros edificios de interés, son líneas rectas. La rectitud es una medida que asume que la gente prefiere

${ }^{13} \mathrm{Krafta}$ (2008) señala que estas medidas de centralidad simple con base en su posición relativa, derivan del trabajo de Freeman (1977) y parte del principio en que una célula $k$ es central para un par de células $i j$, si está interpuesta en su camino más corto. La centralidad es obtenida verificando cuáles individuos promueven la conexión entre cada par de otros y posteriormente, sumando el número total de veces que cada individuo aparece interpuesto en los caminos mínimos entre todos los pares posibles. 
rutas con menos giros, por lo que a mayor rectitud, mayor accesibilidad y mayor centralidad.

Al igual que la medida de Cercanía, la medida de Rectitud es geométrica y puede coadyuvar a entender las lógicas de localización de las piezas de producción y del consumo.

\subsection{Base de datos de unidades económicas de la ciudad de México}

En 2011, el Gobierno de la ciudad de México puso a disposición del público en general, el Sistema de Información Integral (Sinfin), el cual está conformado por una base de datos provenientes tanto de dependencias gubernamentales locales y federales. Alberga registros de casi $417 \mathrm{mil}$ unidades económicas georeferenciadas y descargables en línea mediante los protocolos WMS y wFs. ${ }^{14}$

Este avance en materia de transparencia y acceso a la información permitirá, tanto a investigadores como a agentes privados, analizar diversos fenómenos y apoyar la toma de decisiones tanto en el campo urbano como en materia económica. Para el caso de esta investigación, resulta fundamental el hecho de contar con este banco de datos ya que el análisis de centralidad propuesto parte de datos económicos con alto grado de precisión, situación que hasta hace pocos años era impensable.

\section{Caso de estudio: detección de centralidades en la ciudad de México}

Parece ser que la cuestión sobre la condición policéntrica actual de la ciudad de México ha quedado zanjada. ${ }^{15}$ El debate podrá continuar, pero las evidencias científicas aportadas a favor de la coexistencia de múltiples espacios centrales dejan muy poco lugar a la duda (Ford, 1996; Aguilar y Alvarado, 2005; Garrocho, 1996; Garrocho y Campos, 2007; Graizbord y Acuña, 2005; Graizbord, 2008; Terrazas, 2010; González, 2010).

Los estudios realizados al respecto desde diferentes aproximaciones —la identificación de subcentros de empleo, el análisis de la movilidad partiendo de encuestas origen-destino o el análisis de patrones de actividades estudiados bajo la óptica de la geografía espacio-tiempo- arrojan resultados inequívocos acerca de la condición policéntrica.

\footnotetext{
${ }^{14}$ WFS (Web Feature Services): servicio directo de acceso a datos que permite su lectura, escritura y modificación. wMs (Web Map Services): servicio que permite transformar la información en mapas sin la posibilidad de realizar análisis espacial.

${ }^{15}$ Deben resaltarse que éste es un ejercicio de análisis de centralidad sobre la ciudad de México, no sobre la Zona Metropolitana del Valle de México (ZMVM).
} 
Más aún, la evidencia empírica de cualquier urbanista residente en esta urbe en cuestión, se acercará a la idea en que una buena parte de los habitantes no necesita desplazarse al centro tradicional para satisfacer sus necesidades de consumo inmediatas o desarrollar sus actividades cotidianas. Éstos las pueden realizar en múltiples lugares de la ciudad, en la mayoría de los casos, con el mismo resultado en términos de satisfacción de la actividad realizada o en términos de la calidad del producto adquirido (puede asistir a una red de gimnasios en franquicia o a un centro comercial sin detrimento en el resultado final). Incluso, muchos de los que habitamos esta megalópolis no conocemos determinadas zonas de la ciudad.

Aún con lo anterior, la condición policéntrica de la ciudad de México no es tan evidente. Los fenómenos tanto de conurbación como de metropolización han devenido en un continuo urbano que hace que las centralidades no sean fáciles de identificar a simple vista. Es necesario detectarlas por la presencia de aglomeraciones de actividades económicas o por la presencia de equipamientos de gran escala (centros comerciales o equipamientos educativos) que, en su conjunto, albergan una capacidad intrínseca de atracción de personas.

Más allá sobre la discusión del tipo de subcentros, su tamaño y su grado de especialización — discusión muy relevante pero no desarrollada en esta investigación-, sobre la morfogénesis de los mismos (subcentros especializados o descentralizados) o su condición actual, la presente está focalizada en presentar un método cuantitativo alternativo de medición de centralidades a los ya desarrollados anteriormente.

En todo caso, este estudio pretende, de manera gráfica, presentar el grado de policentricidad que existe en la ciudad y confrontarlo con hallazgos de las investigaciones que han precedido a este intento. En este sentido, debe de advertirse que si bien los resultados parecen ser fiables para el ámbito de estudio (La ciudad de México), no lo son para la realidad metropolitana. Esto es debido a que este ejercicio — que pretende solamente mostrar un método alternativo de medición de centralidadesno incorpora datos sobre toda la Zona Metropolitana del Valle de México, sino solamente los disponibles al momento de hacer esta investigación y que corresponden únicamente al Distrito Federal. Sin embargo, lo relevante de este planteamiento debe de ser el método, más que los propios resultados.

\subsection{Metodología}

El método empleado para para la detección y análisis de centralidades dentro de la ciudad de México puede dividirse grosso modo en tres pasos: 
- La obtención, tratamiento y filtrado de bases de datos y de objetos gráficos a manipular.

- Su análisis mediante algoritmos específicos.

- La interpretación de los resultados y confrontación con hallazgos previos.

Toda la información fue tratada y analizada en un ambiente sIG (ArcGis) mediante herramientas propias del sistema y a través de las herramientas de Análisis de Redes Urbanas (UNA) anteriormente descritas.

\subsection{Obtención, tratamiento y filtrado de datos}

Se partió de dos fuentes de información fundamentales: la base de datos Sistema de Información Integral (Sinfin, 2013) de la Secretaría de Desarrollo Económico del Gobierno del Distrito Federal y la red vial secundaria de la ciudad de México, también obtenida en la misma fuente.

De la base de datos Sinfin, (417 mil registros de unidades económicas), primero se removieron las empresas que tuvieran menos de 11 personas ocupadas, ya que partimos de la base en que una de las principales características de la centralidad urbana es su capacidad de atracción de viajes. Por consiguiente, buscamos las empresas que tuvieran mayor número de empleados, asegurando con ello el análisis de flujos sobresalientes. ${ }^{16}$

Vale la pena señalar que si bien las unidades económicas que emplean de 1 a 10 personas representan aproximadamente cerca de $90 \%$ del total de unidades registradas en la base de datos, éstas muestran un patrón de distribución espacial prácticamente homogéneo en toda la ciudad de México (figura I) por lo que su segregación del presente análisis no altera los resultados de manera significativa. Sin embargo, cabe mencionar que estas unidades económicas destacan por tener presencia en zonas donde no la tienen las unidades económicas mayores a 11 personas ocupadas, específicamente, en las zonas más alejadas del centro tradicional (periferia).

Posteriormente, se aplicó un segundo filtro, eliminando del análisis a la industria, ya que como hemos argumentado, la centralidad debe estar representada por una mezcla de intereses que no aparece en las zonas de uso único (mono-funcionales). Se excluyeron también las actividades relacionadas con la agricultura y la minería, pues su localización se debe al origen mismo de su actividad y no a la búsqueda de sinergias o economías de escala; al comercio al por mayor por representar espacios a los cuales el público en general no suele acudir de manera masiva para ad-

\footnotetext{
${ }^{16}$ Paralelamente a este ejercicio de filtrado de unidades económicas, justificado a lo largo del texto, se corrió el modelo sin filtro de unidades económicas alguno, obteniendo prácticamente los mismos resultados.
} 
quirir sus productos, y al comercio especializado (por ejemplo las tiendas de perfumes o de artículos fotográficos) ya que lo que se intenta detectar son las zonas donde se realizan intercambios de manera intensa en un contexto de cotidianeidad y no de manera esporádica.

\section{Figura I}

\section{Distribución de las unidades económicas por número de empleados en la ciudad de México}

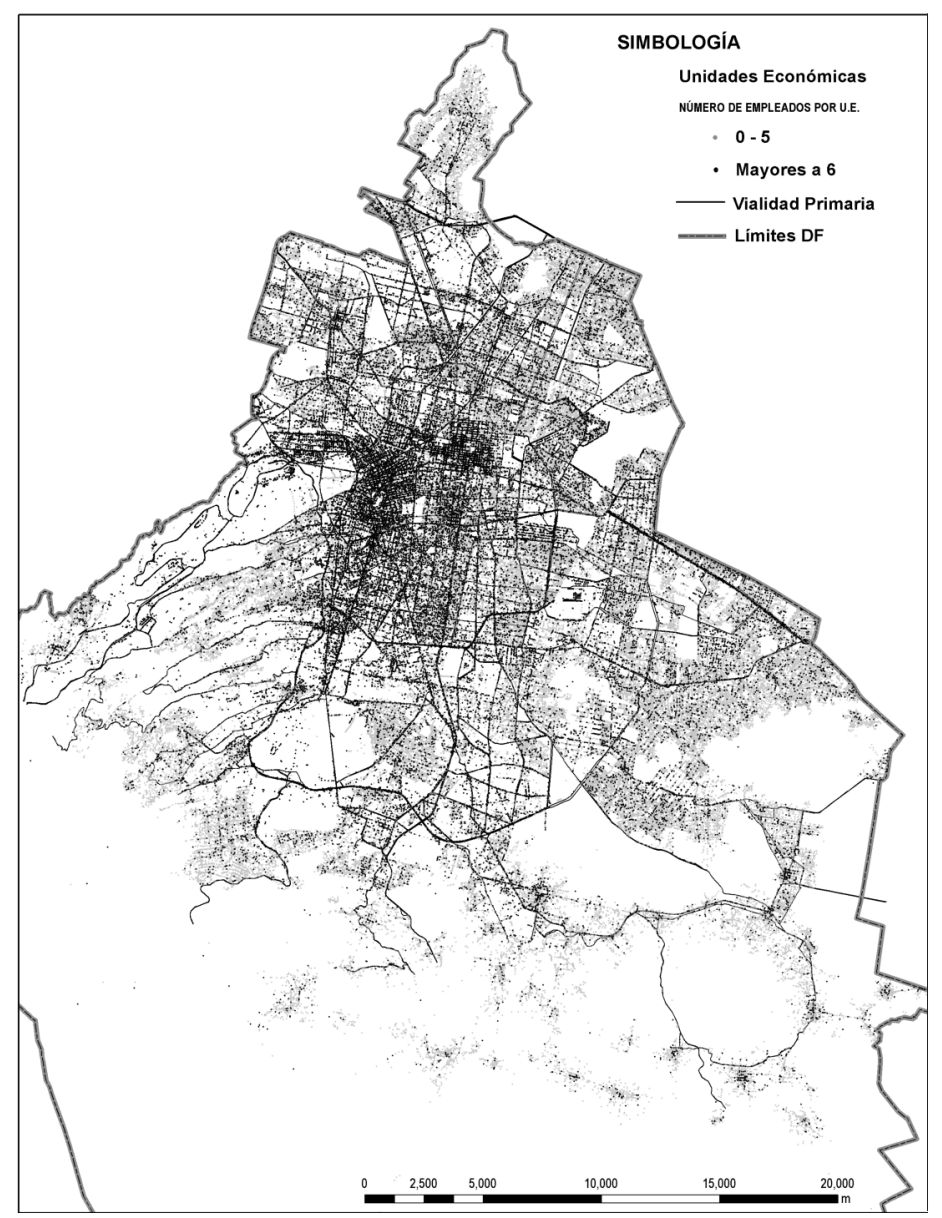

Fuente: elaboración propia con base en Sistema de Información Integral, Gobierno del Distrito Federal (Sinfin, 2013).

La muestra después del filtrado fue de cerca de 25 mil unidades económicas, las cuales concentran $54 \%$ de la población ocupada en unidades económicas mayores a 11 empleados. El restante 46\%, de acuerdo con lo que se puede inferir de esta base de datos, lo hace en unidades económi- 
cas que ocupan a menos de 10 personas distribuidas de manera homogénea en el territorio.

Lo anterior nos permite adelantar unos resultados muy significativos incluso antes de aplicar los algoritmos de Análisis Urbano Espacial (UNA): casi la mitad de la población trabaja en micro y pequeñas empresas las cuales constituyen cerca de $90 \%$ de todas las unidades económicas. La otra mitad, trabaja en empresas medianas y de gran tamaño, que representan tan sólo $10 \%$ de las unidades económicas. Mientras que las primeras se aprecian diseminadas en un patrón homogéneo a todo lo largo y ancho de la ciudad, buena parte de las segundas tienden a concentrarse en la zona central y en corredores específicos.

El siguiente paso previo al análisis espacial fue la preparación informática de la red vial secundaria de la ciudad de México. Debido a errores gráficos, la capa vial pasó por un proceso de limpieza, mediante una función computacional (Split Line) que permitiera el rompimiento de las líneas en todas sus intersecciones, de modo que toda la red estuviera conformada por arcos y nodos.

Luego, la red vial limpia fue transformada en un conjunto de datos de red (Network Dataset), que incorpora un modelo de conectividad avanzado y permite el análisis de redes, como la búsqueda de los caminos más cortos entre nodos a través de los arcos que conforman dicha red, entre otras funciones.

\subsection{Aplicación del Análisis de Redes Urbanas (UNA)}

De las cinco funciones de análisis de redes urbanas desarrolladas por Svetsuk y Mekonnen decidimos aplicar la función de Alcance (Reach). Como se ha señalado, dicha función describe la cantidad de edificios que son alcanzables desde cualquier otro edificio, en la distancia más corta posible dentro de la red vial y con respecto a un radio específico.

Las implicaciones sobre la utilización de esta primera herramienta no son pocas. En primer lugar, se asume que una centralidad está compuesta por una aglomeración de actividades económicas que, debido a la cercanía en sus localizaciones, pueden ser consideradas en muchas ocasiones una sumatoria de clústeres de actividades específicas y complementarias que se asientan a manera de grupo (clúster de clústeres), precisamente buscando la generación de sinergias y economías de escala.

Por otro lado, el hecho de contar ahora con una base de datos tan exacta en materia de localización y orientación de las actividades económicas en la ciudad de México nos permite — sin lugar a dudas_-, resaltar aglomeraciones que, con otro tipo y nivel de desagregación de datos, sería imposible visualizar gráficamente con tanta precisión. 
Si para los análisis de centralidades anteriores el factor clave para su detección era la densidad de empleos en una unidad espacial determinada, ${ }^{17}$ para nuestro modelo, el factor determinante se ha basado no sólo en la concentración de empleo -implícita en la pre-selección de las unidades económicas - sino en la distancia más corta de una localización con respecto a otras en un radio de distancia específico. Dicho radio se ha determinado con base en dos preceptos: en la medida empírica en que una persona está dispuesta a caminar en busca de un satisfactor determinado sin que el desplazamiento implique un obstáculo (gasto de tiempo mayor) que le impida realizar todas sus actividades cotidianas, y en la medida en que la localización de las unidades económicas tiendan a formar clústeres. Ciertamente no existe un consenso para determinar con métodos cuantitativos cuando hay una aglomeración y cuando hay una dispersión. En términos reales, estas categorías varían dependiendo de la escala, y están basados en la relación existente entre todas las unidades económicas que conformen el sistema.

Ante tal indefinición, aparece la medición de la distancia más corta en un radio de $400 \mathrm{~m}$. como una que satisface la cercanía de las actividades en un ámbito pedestre. Probablemente, si estuviéramos midiendo centralidades a nivel metropolitano, el nivel de aglomeración para dictaminar la existencia de un clúster debiera de ser menor, es decir, mesurado con distancias mayores (p.e. $1 \mathrm{Km}$ ). Por otro lado, la determinación de $400 \mathrm{~m}$. en el radio de búsqueda de edificios a la redonda nos aseguró que a escala urbana pudieran ser caracterizadas aglomeraciones que cumplieran con el concepto de centralidad que hemos desarrollado.

Al algoritmo de medición de centralidad de Alcance no se le aplicó ningún índice de impedancia más que la propia distancia del camino más corto al siguiente edificio. Ya que se asumen distancias caminables para la configuración de aglomeraciones de actividades que devengan en centralidades, la impedancia real tendría que ver con los obstáculos que enfrentaría la persona para alcanzar su objetivo (como una banqueta en mal estado, la falta de semáforos en los cruces o la ausencia de rampas para el caso de personas con discapacidad). Para tal efecto hubiera sido necesario catalogar las vialidades por su estado para asignar ese valor y así ponderar el estado de las aceras en el análisis. Del mismo modo, la red vial no fue tratada para simular sentidos viales debido a que al peatón éstos le son indiferentes.

\footnotetext{
${ }^{17}$ Generalmente se usa la hectárea como unidad de medida espacial. El problema de las mediciones anteriores no radica en la consistencia de la unidad de medida, sino que ésta a su vez se relaciona con un ámbito espacial político (municipio, delegación o división comarcal), que presenta grandes diferencias de tamańo. Se comparan pues, densidades de empleo con relación al área de límites administrativos dispares.
} 
Con los mismos datos se realizaron dos cómputos distintos: uno sin ponderar el número de empleados y otro ponderándolos. Mientras que el primer cálculo mostraría el número de unidades económicas alcanzables para todas en un radio de $400 \mathrm{~m}$ el segundo calcularía el número de empleados alcanzables para cada unidad económica en un radio igual.

\section{Figura II}

\section{Centralidades urbanas: unidades económicas en un radio de $400 \mathrm{~m}$}

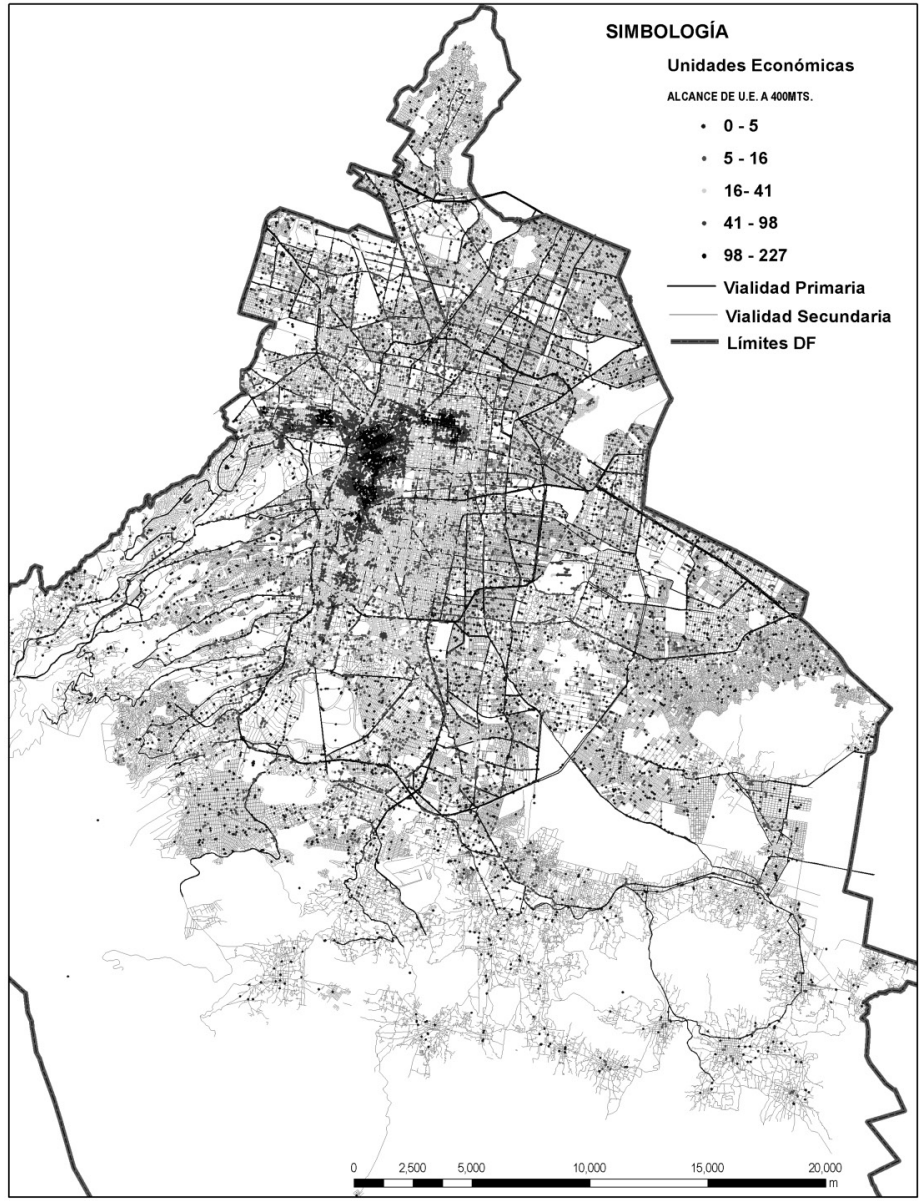

Fuente: elaboración propia con base en el Sistema de Información Integral, Gobierno del Distrito Federal (Sinfin, 2013). 


\section{Figura III}

\section{Centralidades urbanas: empleos en un radio de $400 \mathrm{~m}$}

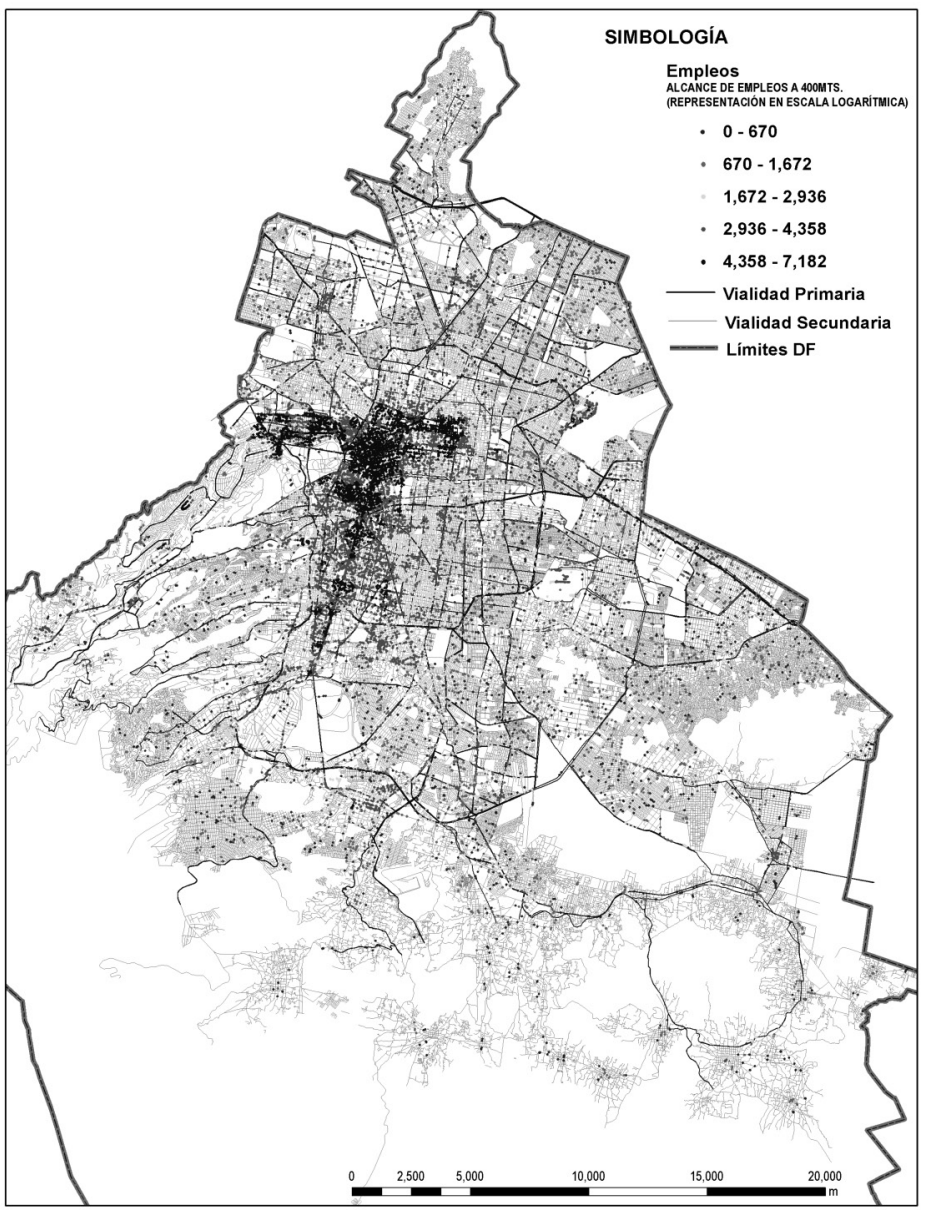

Fuente: elaboración propia con base en el Sistema de Información Integral, Gobierno del Distrito Federal (Sinfin, 2013).

\section{Resultados}

La figura II muestra las 25 mil unidades económicas geo referenciadas, agrupadas en cinco distintos rangos, jerarquizadas por el número de unidades económicas que cada una de ellas encontró por el camino más corto en un radio de $400 \mathrm{~m}$.

En su conjunto, se aprecia claramente una concentración de actividades mucho más acusada en la parte central de la ciudad que en los linderos de los límites administrativos. Ésta aglomeración central de actividades está conformada por distintos gradientes que se acentúan sobre el corredor de 
Insurgentes — desde Copilco hasta Santa María la Ribera (eje norte-sur) — y sobre el corredor que inicia en Lomas de Chapultepec y culmina en el Centro Histórico (eje poniente-oriente), formando una especie de cruceta.

Se aprecian también, a nivel general, aglomeraciones de menor intensidad (o en proceso de consolidación) a lo largo del corredor de Av. Revolución, con tendencia a su difuminación hacia el poniente, mientras que se avizora otra importante concentración lineal de actividades a lo largo de Av. Universidad y de División del Norte.

Estudiando más a fondo esta aglomeración central, queda demostrada la aportación de Terrazas (2010: 9): "Sostengo que la localización de la centralidad corresponde a un patrón de ejes o caminos, representados estos por las principales vías de comunicación y rutas de transporte en el interior de la ciudad". Sin embargo, también es perfectamente visible la formación de aglomeraciones en entramados viales constituidos por arterias secundarias, hecho que aumenta a identificar simbólica y espacialmente a las unidades barriales más cercanas al conocimiento de la gente: las colonias.

Uno de los problemas a los que nos enfrentamos para detectar centralidades bajo esta metodología es que la figura II muestra los gradientes de resultados sin ningún tipo de normalización. Esto hace más difícil la identificación de los subcentros a primera vista, pues en los métodos ya citados, la normalización de los resultados mediante la densidad de empleo desempeña un papel importante para su detección.

En nuestro caso, prescindimos de esa normalización por densidad de empleo pues — como se ha señalado_-, parte de ámbitos geopolíticos dispares, además de que el objetivo de este método es precisamente mostrar el fenómeno de la aglomeración lo más realista posible. Al comparar rangos tan alejados en términos de valores para un mismo sistema (lo que per se indica claramente una situación de hiperconcentración), nos hemos valido de la normalización logarítmica para su representación (figura III), de tal modo que las centralidades de menor jerarquía sean apreciables a simple vista ante la potencia de la aglomeración central.

\subsection{Centralidades en la ciudad interior}

De la gran aglomeración central de actividades anteriormente mencionada, es posible destacar subcentros que se encuentran en condición de contigüidad con los demás grupos de actividades pero que destacan por rango.

Entre las de primer orden se encuentran las colonias Lomas de Chapultepec, Polanco, Anzures, Cuauhtémoc, Juárez, Tabacalera, Roma norte, Hipódromo, Hipódromo Condesa, Escandón, Centro, San Pedro de los Pinos, el cruce de Insurgentes-Mixcoac y la Guadalupe Inn. 
Entre las de segundo orden destacan: Polanco tercera sección, Verónica Anzures, San Rafael Granada, Obrera, Condesa, Roma sur, Narvarte poniente, Tacubaya, Nápoles, Del Valle norte, Tacubaya, San Ángel, Coyoacán, Copilco y el Barrio de Loreto.

Como hemos señalado, varias de las centralidades se han ido desarrollando a partir de las principales vías de comunicación, destacando Insurgentes y Revolución como grandes ejes de ordenamiento de las actividades económicas. También destacan ejes como Cuauhtémoc, Universidad y División del Norte. Es de resaltar que el Periférico, a excepción de algunos cruces importantes (con Reforma, por ejemplo), no parece tener la misma capacidad de atracción de actividades que cualquier otra de las vialidades mencionadas.

\subsection{Centralidades en la ciudad extendida}

Le denomino para este caso de estudio ciudad extendida a las áreas donde existen centralidades de primero y segundo orden pero que no guardan contigüidad física con la aglomeración central de actividades anteriormente descrita. Con ello eludí conscientemente en este estudio el debate sobre la taxonomía del crecimiento urbano de la ciudad de México.

Dentro de los grandes equipamientos, se puede destacar la presencia de la Central de Abastos como un subcentro de empleo. También debería destacarse Ciudad Universitaria pero, debe señalarse, este método de análisis falla en el sentido en que no es capaz de detectar centralidades cuando no existen aglomeraciones de actividades, a menos que el valor sea ponderado por algún indicador específico (por ejemplo, el número de personas ocupadas). A pesar de ello, en Copilco (en las inmediaciones de $\mathrm{CU}$ ) se ha detectado una centralidad debido a la alta concentración de servicios orientados a la educación (fotocopiadoras, impresores, restaurantes, comercios, etc.).

Por otro lado, solamente se detectó una centralidad de primer orden, desligada de la aglomeración central, ubicada dentro de Bosques de las Lomas. Tal pareciera que Santa Fe debiera destacar como una centralidad de primer orden, pero muy probablemente los datos de donde emana el análisis —obtenidos al 2011 — no estén reflejando los más de $30 \mathrm{mil}$ viajes diarios que se realizan a esa zona de la ciudad o que efectivamente, no tenga la misma capacidad de atracción bajo los parámetros en que se analizó al sistema en su conjunto.

Como centralidades de segundo orden, dispersas en la ciudad extendida, aparecen además de Santa Fe y Paseos de las Lomas, Cuajimalpa, Tlalpan, Los Girasoles (Coapa), Peñón de los Baños, GuadalupeTepeyac, 
Azcapotzalco y Lindavista (zona de inserción del Instituto Politécnico Nacional).

Aun cuando en el análisis no alcanzaron la categoría de centralidades de segundo orden, existen algunas aglomeraciones que parecen formar parte del sistema parcialmente policéntrico de la ciudad de México, como el Barrio de San Antonio en Xochimilco, el Barrio de la Asunción en Tláhuac o, en un nivel muy inferior de categoría, el Barrio de San Mateo en Milpa Alta.

\section{Conclusiones}

Ciertamente, la condición policéntrica de la ciudad de México existe. Sin embargo, no es tan evidente como estudios anteriores lo han señalado (Garrocho, 1996) y, sin tomar como unidad de análisis al sistema metropolitano en su conjunto, es posible afirmar que esta metropoli no ha pasado por un proceso de dispersión/reconcentración similar al caso norteamericano (edge cities). La estructura de la ciudad presenta todavía una muy fuerte concentración de actividades y densidad de empleo en la zona central con respecto al resto del sistema analizado.

Dentro de los límites administrativos es factible concluir que se aprecia una estructura polinuclear, con un centro altamente dominante y subcentros de empleo en proceso de consolidación. A pesar de coincidir, tanto en resultados generales como en la apreciación sobre la condición del grado de policentrismo de Suárez y Delgado (2009), el análisis aquí presentado muestra también una red de unidades económicas dispersas de manera homogénea en el territorio que, si bien no conforman centralidades, sí eliminan la necesidad de tener que acudir a la gran aglomeración central para casi la mitad de las personas ocupadas. Así, nos enfrentamos con un problema mucho más complejo en términos de modelo, pues se superponen como capas bien diferenciadas pero yuxtapuestas, la concentración y la dispersión de actividades.

Probablemente el hallazgo más importante en términos de resultados es, que mediante este método, se llegó a conclusiones muy similares a las planteadas por Suárez y Delgado (2009) y por González (2010) para sus respectivos estudios. Las debilidades detectadas que exhibe este estudio de caso son claras: no se presentan resultados a nivel metropolitano. Esta primera aproximación buscó validar el método como uno confiable, por lo que el siguiente paso será reproducir este método con datos del Directorio Estadístico Nacional de Unidades Económicas (DENUE) para toda la Zona Metropolitana del Valle de México (zMvm).

Aún con las limitaciones señaladas, queda evidenciada la potencia que este método tiene para el análisis de sistemas urbanos, el cual deberá de 
irse perfeccionando a la par que los datos para el área metropolitana puedan ser incorporados en los mismos formatos. Se ha llegado a un resultado que nos ha permitido la caracterización de los espacios centrales sin necesidad de la utilización de los sistemas tradicionales o sin cruzar la información con la población residente. Se desprende de ello que este método podría complejizarse para poder brindar información certera a los tomadores de decisiones sobre las acciones de planeamiento futuro.

\section{Bibliografía}

Aguilar, Adrián y Concepción Alvarado (2005), “La reestructuración del espacio urbano de la ciudad de México. Hacia la metrópoli multimodal" en Adrián Aguilar (ed.), Procesos metropolitanos y grandes ciudades: Dinámicas recientes en México y otros países, Colegio de México-Cámara de Diputados-Universidad Nacional Autónoma de México-Consejo Nacional de Ciencia y Tecnología-Miguel Ángel Porrúa, México, pp. 265-308.

Allen, Peter (1997), Cities and regions as self-organizing systems: models of complexity, Taylor \& Francis, Londres.

Alonso, William (1964), Location and land use; toward a general theory of land rent, Harvard University Press, Cambridge.

Batty, Michael (2001), "Polynucleated urban landscapes", Urban Studies, 38 (4), Sage Journals, London, pp. 635-655.

Bogart, William y William Ferry (1999), "Employment centres in greater cleveland: evidence of evolution in a formerly monocentric city", Urban Studies, 36 (12), Sage Journals, London, pp. 2099-2110.

Camagni, Roberto (2005), Economía urbana, Antoni Bosch, Barcelona.

Casado, Ignacio (2010), “Apuntes para la delimitación y estudio de un centro urbano", Eumed.net, <http://www.eumed.net/rev/ cccss/07/icg.htm>, 7 de marzo del 2010.

Castells, Manuel (1974), La cuestión urbana, Siglo xxi Editores, México.

Champion, Anthony (2001), "A changing demographic regime and evolving poly centric urban regions: consequences for the size, composition and distribution of city populations", Urban Studies, 38 (4), Sage Journals, London, pp. 657-677. 
Clark, William y Marianne Kuijpers-Linde (1994), "Commuting in restructuring urban regions", Urban Studies, 31 (3), Sage Journals, London, pp. 465-483.

Dematteis, Giuseppe (1994), Il Fenomeno urbano in Italia : interpretazioni, prospettive, politiche, Franco Angeli, Milano.

Ford, Larry (1996), "A new and improved model of Latin American City structure", Geographical Review, 86 (3), Latin American Geography, New York, pp. 437-440.

Freeman, Linton (1977), "A set of measures of centrality based on betweenness", Sociometry, 40 (1), American Sociological Association, Washington, pp. 35-41.

Fujita, Masahisa y Tomoya Mori (1997), "Structural stability and evolution of urban systems", Regional Science and Urban Economics, 27 (4), Elsevier, Netherlands, pp. 399-442.

Fujita, Masahisa, Paul Krugman y Anthony Venables (2001), The spatial economy: cities, regions and international trade, MIT Press, Cambridge.

García-López, Miquel Ángel e Iván Muñiz (2010), “Employment decentralisation: polycentricity or scatteration? the case of Barcelona", Urban Studies, 47 (14), Sage Journals, London, pp. 3035-3056.

Garrocho, Carlos (1996), "Distribución espacial de la población en la Zona Metropolitana de la Ciudad de México, 1950-1990", Estudios Demográficos y Urbanos, 11 (1), El Colegio de México, México, pp. 69-100.

Garrocho, Carlos y Juan Campos (2007), “Dinámica de la estructura policéntrica del empleo terciario en el área metropolitana de Toluca, 1994-2004", Papeles de Población, 13 (52), Universidad Autónoma del Estado de México, Toluca, pp. 109-135.

Giuliano, Genevieve y Kenneth Small (1991), "Subcenters in the Los Angeles region”, Regional Science-Urban Economics, 21 (2), Elsevier, Washington, pp. 163-182. 
González, Salomón (2010), "Policentralidad a partir de los patrones viaje -actividad en la ZMvм", en Óscar Terrazas (ed.), La ciudad que hoy es centro, Universidad Autónoma Metropolitana unidad Azcapotzalco, Consejo Nacional de Ciencia y Tecnología, México, pp. 27-52.

Graizbord, Boris y Beatriz Acuña (2005), "La estructura polinuclear del área metropolitana”, en Adrián Aguilar (ed.), Procesos metropolitanos y grandes ciudades. Dinámicas recientes en México y otros paises, Cámara de Diputados-Universidad Nacional Autónoma de México-Consejo Nacional de Ciencia y Tecnología-Miguel Ángel Porrúa, México, pp. 309-328.

Graizbord, Boris (2008), Geografía del transporte en el área metropolitana de la ciudad de México, Colegio de México-Centro de Estudios Demográficos, Urbanos y Ambientales, México.

Haig, Robert (1926), "Toward an understanding of the Metropolis: II. The assignment of activities to areas in urban regions", The Quarterly Journal of Economics, 40 (3), Oxford University Press, Oxford, pp. 402-434.

Hall, Peter (1997), "Modelling the post-industrial city", Future, 29 (4-5), Elsevier, pp. 311-322.

Hall, Peter y Kathy Pain (2006) (eds.), The polycentric metropolis learning from mega-city regions in Europe, Earthscan Publications, Sterling, London.

Hansen, Walter (1959), "How accessibility shapes land use", Journal of the American Institute of Planners, 25 (2), pp. 73-76, <http://www. tandfonline.com/doi/pdf/10.1080/01944365908978307>, $8 \mathrm{de}$ diciembre de 2014.

Hurd, Richard (1905), Principles of city land values, The Record and guide, Nueva York.

Krafta, Romulo (2008), "Fundamentos del análisis de centralidad espacial urbana”, Centro- $h$, (2), Organización Latinoamericana y del Caribe de Centros Históricos, Ecuador, pp. 57-72. 
Kunz, Ignacio (2003), Usos de suelo y territorio: tipos de lógicas de localización en la ciudad de México, Plaza y Valdés, México.

McDonald, John (1987), "The identification of urban employment subcenters", Journal of Urban Economics, 21 (2), Elsevier, Washington, pp. 242-258.

Méndez, Ricardo e Inmaculada Caravaca (1996), Organización industrial y territorio, Síntesis, Madrid.

Mercado, Alejandro y María Moreno (2011), La ciudad de México y sus clusters, Universidad Autónoma Metropolitana, México.

Muñiz, Iván, Ana Galindo y Miguel García (2005), "Descentralización, integración y policentrismo en Barcelona”, working paper, Universitat Autònoma de Barcelona, Barcelona, <http://www.ecap. uab.es/repec/doc/wpdea0505.pdf>, 7 de marzo del 2013.

Okabe, Atsuyuki, Kei-Ichi Okunuki y Shino Shiode (2006), “The Sanet toolbox: new methods for network spatial analysis", Transactions in GIS, 10 (4), Wiley Online Library, pp. 535-550.

Paris, Mario (2013), "De los centros urbanos consolidados a los lugares de centralidad: una propuesta metodológica para su estudio", Ciudades, 16 (1), Universidad de Valladolid, Valladolid, pp. 47-69.

Porter, Michael (1998), "Clusters and the new economics of competition", Harvard Business Review, 76 (6), Harvard Business Publishing, Boston, pp. 77-90.

Sinfin (Sistema de Información Integral) (2013), "Centralidades urbanas, Gobierno del Distrito Federal”, <www.sinfin.gob.df.mx>, 7 de marzo del 2013.

Sevtsuk, Andrés y Michael Mekonnen (2001), "Urban network analysis: a new toolbox for ArcGIS”, working paper, MIT, Cambridge, <http://cityform.mit.edu/papers.html>, 7 de marzo del 2013.

Storper, Michael y Anthony Venables (2002), "Buzz: the economic force of the city", ponencia presentada en la conferencia Industrial Dynamics of the New and Old Economy -- who is embracing whom, 6-8 de junio, Copenhagen/Elsinore, Dinamarca. 
Suárez, Manuel y Javier Delgado (2009), "Is Mexico city polycentric? A trip attraction capacity approach", Urban Studies, 46 (10), Sage Journals, London, pp. 2187-2211.

Terrazas, Óscar (2004), "La centralidad metropolitana en la ciudad de México", en Ariel Rodríguez y Sergio Tamayo (eds.), Los últimos cien años, los próximos cien..., Universidad Autónoma Metropolitana, México, pp. 235-265.

Terrazas, Óscar (2010) (ed.), La ciudad que hoy es centro, Universidad Autónoma Metropolitana unidad Azcapotzalco, Consejo Nacional de Ciencia y Tecnología, México.

Thurstain-Goodwin, Mark y David Unwin (2000), "Defining and delineating the central areas of towns for statistical monitoring using continuous surface representations", Transactions in GIS, 4 (4), Blackwell Publishers Ltd, Massachusetts, pp. 305-317.

Weber, Alfred y Carl Friedrich (1929), Alfred Weber's theory of the location of industries, The University of Chicago Press, Chicago.

White, Michelle (1999), "Urban areas with decentralized employment: Theory and empirical work", Handbook of Regional and Urban economics, 3, Elsevier, Netherlands, pp. 1375-1412.

Recibido: 14/01/2013.

Reenviado: 29/037/2013.

Aceptado: 19/9/2013.

Jorge Alberto Montejano-Escamilla. Mexicano. Es doctor en urbanismo por la Universitat Politécnica de Catalunya (Tesis Excelente Cum-Laude), Arquitecto por la Universidad Iberoamericana. Actualmente es profesorinvestigador titular "B" en el Centro de Investigación en Geografía y Geomática "Ing. Jorge L. Tamayo", A. c. (Centrogeo). Sus líneas actuales de investigación son metropolización territorial, movilidad, vivienda, lógicas y modelos de localización y sIG aplicados a estudios urbanos. Entre sus publicaciones destacan: "El impacto de las nuevas tecnologías en la 'explosión' de la ciudad”, URBS. Revista de Estudios Urbanos y Ciencias Sociales, 3 (1), Universidad de Almería, Almería, pp. 45-67 (2013); en coautoría: "Hacia barrios autocontenibles en la Zona Metropolitana del Valle de México", Revista Iberoamericana de Urbanismo, 10, riURB_ 
Editores-Universitat Politècnica de Catalunya, Buenos Aires-Palma de Mallorca-Barcelona, pp. 53-72 (2013); "Nuevos procesos de metropolización del territorio", Espacialidades: Revista de temas contemporáneos sobre lugares, politica y cultura, (3) 2, Universidad Autónoma Metropolitana unidad Cuajimalpa, México, pp. 34-66 (2013); "Neourbanismo y la Zona Metropolitana del Valle de México", Ciencia y Desarrollo, 39 (267), Conacyt, México, pp. 38-42 (2013); "Lo que queda (Expo Zaragoza 2008)”, la Tempestad, 63, México, pp. 68-72 (2008). 